\title{
A FORMAÇÃO DO CUIDADOR DE IDOSOS INSTITUCIONALIZADOS: ENFASE NA ROTINA DE ALIMENTAÇÃO
}

\author{
Jáima Pinheiro de Oliveira ${ }^{1}$ \\ Juliana Ferreira Marcolino² \\ Michelly Santos de Andrade ${ }^{3}$
}

resumo

Este estudo objetivou discutir sobre o perfil do cuidador de idosos institucionalizados para auxiliar na tarefa de alimentação. Foram feitas entrevistas com cinco profissionais da saúde, atuantes em uma Instituição de Longa Permanência para idosos (ILPIs). No estudo do perfil do cuidador de idosos institucionalizados, para auxiliar na tarefa de alimentação, verificou-se que os cuidadores relataram fatores de

1 Fonoaudióloga; Mestre em Educação Especial pela Universidade Federal de São Carlos (UFSCar/ SP); Doutoranda em Educação pela Faculdade de Filosofia e Ciências da Universidade Estadual Paulista (UNESP/SP); Docente do Departamento de Fonoaudiologia da Universidade Estadual do CentroOeste (UNICENTRO/PR), Campus de Irati/PR. E-mail: jaimafono@gmail.com

2 Fonoaudióloga, Mestre e doutoranda em Linguística Aplicada e Estudos da Linguagem pela PUCSP; Docente do curso de Fonoaudiologia da Universidade Estadual do Centro-Oeste (UNICENTRO/ PR). E-mail: jumarcolino@hotmail.com

3 Fonoaudióloga, Mestre em Saúde Coletiva pela Universidade Federal de Pernambuco (UFPE); Docente do curso de Fonoaudiologia da Universidade Estadual do Centro-Oeste (UNICENTRO/PR). E-mail: mandradefono@gmail.com 
risco no que tange modificações da deglutição e a não realização da a higiene oral dos sujeitos. Nossos resultados sugerem a necessidade de reflexões sobre a formação desses cuidadores para auxiliar na alimentação dos idosos. O fonoaudiólogo poderá orientar os cuidadores a fim de facilitar a deglutição dos idosos em ILP.

palavras-chave

Cuidadores. Instituição de Longa Permanência para Idosos.

Alimentação. Formação.

\section{Introdução: o envelhecimento e o processo de institucionalização}

O número crescente de idosos dependentes de instituição de longa permanência (ILP) levanta diversas questões relevantes. Deve-se, primeiramente, indagar sobre os motivos da necessária institucionalização dos idosos. A dependência, o abandono e a falta de cuidados podem ser alguns dos motivos da internação, o que poderia caracterizar o perfil do idoso em ILP no Brasil. Por outro lado, se o idoso institucionalizado possui maior dependência, deve-se questionar como os profissionais dessas instituições estão preparados para as mais diversas funções exigidas nesse cotidiano. Como as tarefas dos cuidadores/profissionais da saúde que trabalham nas instituições são amplas e com diferentes focos de trabalho, elegemos, neste artigo, a tarefa de auxílio à alimentação do idoso.

Atualmente, a maior parte dos estudos sobre a população idosa mostra um crescimento dessa faixa etária de forma mais acentuada nos países em desenvolvimento, o que implica indiscutivelmente na necessidade de aprimoramento, quando não de criação, de políticas públicas de atenção para a terceira idade (BRASIL, 2007). Nesse sentido, alguns estudos epidemiológicos têm indicado que doenças e limitações não são consequências inevitáveis do envelhecimento e, portanto, o uso de serviços preventivos, no sentido de eliminar fatores de risco e adotar hábitos de vida saudáveis são importantes determinantes do envelhecimento saudável (LIMA-COSTA; BARRETO, 2003; KANNEL, 1997; DESAI; ZHANG).

Por outro lado, estudos como o de Mendonça e Marques Neto (2003) e Ramos et al. (1993) mostram que a maioria dos idosos tem, pelo menos, uma doença crônica. Isso quer dizer que o envelhecimento saudável, de fato, não 
pode ser concebido como ausência de doenças crônicas. A partir do novo paradigma de saúde, é a capacidade funcional que determina a saúde no envelhecimento. Em outras palavras, é a autonomia do idoso que joga um papel decisivo nessa questão. Segundo Ramos (2003), o envelhecimento saudável é o resultado de um equilíbrio de diversas dimensões (fisica, psíquica, econômica, social) que permite ao idoso ter independência para administrar todos os problemas existentes nessas dimensões.

Vê-se, portanto, que a manutenção da capacidade funcional do idoso é um desafio para o sistema de saúde. Prova disso, é que gerontólogos e geriatras têm se deparado, na clínica, com a síndrome da fragilidade, caracterizada por "fraqueza muscular, fadiga e baixa tolerância aos estressores físicos e psicológicos" (TEIXEIRA; NERI, 2006). Desse modo, a fragilidade seria um declínio funcional que coloca o sujeito numa situação vulnerável, por exemplo, à institucionalização e à incapacidade (GOLDFARB, 2006).

Ressalta-se a distinção entre as palavras fragilidade e incapacidade. A situação de fragilidade é heterogênea e não atinge todos os idosos da mesma forma, pois depende do declínio biológico e de fatores sociais, subjetivos e culturais. A incapacidade é um desfecho possível da fragilidade, indicando restrições e limitações das atividades (OMS, 2003).

Outra questão bastante importante e que ainda gera muita polêmica é a institucionalização dos idosos. Acredita-se que o sujeito institucionalizado não concebe a velhice da mesma maneira do idoso não-institucionalizado. A pesquisa de Oliveira e Santos (2008), realizada em uma instituição asilar chamou a nossa atenção para o fato de que a maioria dos idosos relatou o desejo de ir embora da instituição e, até mesmo, desconsiderou a existência de membros da família. O que as autoras encontraram nos relatos desses sujeitos foi um desprazer por ser velho institucionalizado.

Os estudos de Mendonça e Marques Neto (2003) com grupos de idosos institucionalizados indicam que dentre os fatores de risco para esse processo de internamento encontram-se as síndromes de imobilidade, problemas médicos, depressão, demência, idade acima dos 70 anos, estado civil solteiro, não possuir filhos, morar sozinho, isolamento social e pobreza. Seguindo tais pressupostos, alguns estudos têm confirmado que os problemas de saúde e a consequente perda de autonomia não surgem como os principais fatores apontados pelos idosos para a decisão do internamento. Na verdade, essa decisão pelo internamento nas Instituições de Longa Permanência (ILP) está voltada para o fato deles não terem uma rede de interações que facilitem a integração social e familiar (FREIRE; TAVARES, 2005). 
A fase da vida em que o idoso entra para uma instituição é outro fator a ser considerado. Essa fase, normalmente, é representada como a última etapa de sua vida, isto é, sem expectativa de retorno. Dessa forma, esse processo de internamento parece se tornar uma situação irreversível e, provavelmente por isso, alguns autores consideram tal processo como o abandono, o sofrimento e a morte (GOLDFARB, 2006).

Nesse sentido, embora nas Instituições de Longa Permanência sejam mantidos convívios sociais, fundamentais para a manutenção do senso de pertinência do ser humano, a condição de institucionalizar-se implica em perda de autonomia para o idoso. Isso, por sua vez, o exclui do trabalho, das funções de aquisição de produção, manutenção e transmissão de conhecimentos. Sendo assim, não será difícil de prever que, nessas circunstâncias, esse idoso tenha tendência a se isolar, assumindo em muitas vezes, uma situação de dependência (KARSCH, 2003; OLIVEIRA; SANTOS, 2008). Essa nova realidade traz à tona questões como a moradia e o cuidado e quais as responsabilidades dos governos, da sociedade e da família com relação a elas (BORN, 2001).

Sobre essas instituições que fornecem atendimento à terceira idade, o Estatuto do Idoso (BRASIL, 2007) preconiza que essas entidades deverão ter condições físicas adequadas, fornecer vestuários, alimentação suficiente, proporcionar atividades esportivas, de lazer, culturais e recursos humanos com formação específica para esse atendimento. O que se vê na prática, muitas vezes, é o cuidador como o único responsável pelos idosos que ali residem. Na maioria dos casos, é ele que representa o vínculo do idoso com o outro, já que todos os outros laços sociais foram desarranjados. Desse modo, esses cuidadores, além de assumirem um perfil próprio, são submetidos às mais diversas condições de desgastes físico e emocional (ZARIT ${ }^{4}$ apud CERQUEIRA; OLIVEIRA, 2002).

Para que essa situação fosse modificada, seria necessário que grande parte dessas instituições passasse por um processo de reestruturação, a fim de cumprir, de maneira satisfatória, suas responsabilidades implícitas na legislação. Essas responsabilidades seriam de proporcionar um lar, um lugar de vida, de aconchego, de identidade e, ao mesmo tempo, de cuidados. Alguns estudos já mostram que essas mudanças são possíveis (MARTINS; MASSAROLLO, 2008).

4 ZARIT, Steven. Interventions with family caregivers. In: ZARIT, Steven; KNIGHT, Bob G. (Eds.). A guide to Psychotherapy and Aging. Washington, DC: American Psychological Association, p. 139-159, 1997. 
Em todo esse contexto de institucionalização do idoso, o cuidador é alguém que assume um papel fundamental de ajudar o idoso em diversas tarefas, tais como: higiene, fazer compras, cuidar das finanças, controlar a medicação, administrar a alimentação, limpar a casa. No Brasil, geralmente, é a família, os chamados "cuidadores informais", que assume esse papel. Em outros casos, os idosos são assistidos por um profissional remunerado (GONÇALVES, 2002).

Acredita-se que o cuidador formal do idoso institucionalizado é um profissional com formação especializada devido à variedade de conhecimentos exigidos. Entretanto, pouco se conhece sobre o perfil e a formação do profissional (SALIBA et al., 2007). Nesse sentido, há urgência em construir um conhecimento multiprofissional para qualificar o cuidador e promover melhor qualidade de vida ao idoso. A Universidade pode ser mais um dispositivo para veicular esse conhecimento e refletir sobre a atuação desse profissional.

Um dos focos dessa atuação deve ser o auxílio na alimentação. Nessa tarefa, o cuidador deverá posicionar o sujeito, fornecendo os utensílios, volume e consistência dos alimentos, principalmente, nos casos em que o idoso apresente dificuldades de deglutição.

A deglutição é o ato de conduzir o alimento da cavidade oral em direção ao estômago, não permitindo entrada de material nas vias aéreas (MARCHESAN, 2004). Pode-se acrescentar, ainda, a importância da deglutição em suprir as necessidades nutricionais diárias, proporcionando também prazer durante a alimentação e, em algumas situações, apresentando o caráter social.

Rockland e Borba (2006) afirmam que as dificuldades da passagem de alimentos da cavidade oral para o estômago podem ser divididas quanto a sua etiologia no caso de idosos. Têm-se, então, os efeitos primários que são aqueles decorrentes do próprio processo de envelhecimento e, os efeitos secundários, oriundos de enfermidades do idoso, tais como: AVE, Esclerose Lateral Amiotrófica, Doença de Parkinson, Doença de Alzheimer, entre outras. E, por fim têm-se os efeitos terciários que são as alterações relacionadas aos fatores ambientais, sociais e emocionais.

A mudança da deglutição, decorrentes do processo de envelhecimento, recebe o nome de presbifagia. Alguns fatores podem agravar essa situação, como por exemplo, o uso de próteses dentárias mal adaptadas (JALES et al., 2005) e, a presença de algumas doenças comuns no processo de envelhecimento. 
O conhecimento acerca desse processo e de possíveis fatores que interferem nele são de fundamental importância para um cuidado satisfatório em relação à alimentação do idoso. Por isso, nesse estudo enfatizamos tal aspecto, por ser o fonoaudiólogo o profissional capacitado para desenvolver a prevenção, o diagnóstico e o tratamento dos distúrbios de deglutição orofaríngeos. Em função disso, talvez seja este o mais preparado para formar o cuidador sobre esse tema.

Levando-se tais pressupostos em consideração, este estudo objetivou discutir sobre o perfil do cuidador de idosos institucionalizados para auxiliar na tarefa de alimentação.

\section{Método}

O presente estudo caracteriza-se como do tipo descritivo-exploratório, a partir da análise de entrevista com $05^{5}$ profissionais da saúde, com idades entre 31 e 45 anos, atuantes em uma Instituição de Longa Permanência (ILP) de uma cidade do interior do Estado do Paraná.

A coleta dos dados aconteceu depois da autorização por escrito da direção do local supracitado. O início das entrevistas se deu após o esclarecimento dos objetivos da pesquisa resultando na assinatura do Termo de Consentimento Livre e Esclarecido (TCLE) de cada um desses cuidadores. Para a realização da entrevista, foi utilizado um roteiro semiestruturado, contendo questões relativas aos cuidados realizados no cotidiano da instituição, com ênfase para a rotina alimentar.

Para melhor análise dos relatos obtidos na entrevista, optou-se pela técnica de transcrição. Para isso, as mesmas foram gravadas utilizando-se um gravador digital Olympus DVR VN -120. Nessa análise, procurou-se contemplar, por meio de categorização temática, aspectos de: perfil dos cuidadores; cuidados gerais e práticas de lazer da instituição; fatores de risco para alterações de deglutição em idosos; posicionamento durante a alimentação; e práticas de higienização oral. Os resultados foram dispostos em quadros e tabelas e discutidos à luz da literatura.

\footnotetext{
5 O n deste estudo representa a realidade da região estudada. Há uma ILP com quase 46 idosos internados e 5 profissionais de saúde atuando nesta instituição. Desse modo, o $n=5$, apesar de necessário, não foi ampliado, porque caracteriza várias instituições do interior do Paraná.
} 
Quadro 1 - perfil dos cuidadores

\begin{tabular}{|c|c|c|c|c|}
\hline Participantes & Sexo & Idade & Formação & $\begin{array}{c}\text { Tempo de } \\
\text { atuação com } \\
\text { idosos }\end{array}$ \\
\hline P1 & F & 34 & Auxiliar de enfermagem & 2 anos \\
\hline P2 & F & 38 & $\begin{array}{c}\text { Técnica em enferma- } \\
\text { gem }\end{array}$ & 8 anos \\
\hline P3 & F & 41 & Auxiliar de enfermagem & 6 anos \\
\hline P4 & F & 35 & Auxiliar de enfermagem & 4 anos \\
\hline P5 & F & 45 & Auxiliar de enfermagem & 10 anos \\
\hline
\end{tabular}

Chama a atenção o predomínio do sexo feminino na população estudada. A literatura afirma que esse fato deve-se ao papel culturalmente desempenhado pela mulher de cuidar da família, da casa, dos filhos e parentes (SALIBA et al., 2007). Desse modo, não é incomum encontrarmos cuidadores domiciliares que, além da atenção dada ao idoso, também fazem serviços domésticos, o que implica em cuidados não satisfatórios dispensados ao idoso (KAWASAKI; DIOGO, 2001).

Observa-se no Quadro 1, que todos os cuidadores possuem formação na área de enfermagem, sendo a maioria em nível técnico. Saliba et al. (2007) afirmam que raros são os trabalhos que descrevem o perfil dos cuidadores formais. Além disso, os autores enfatizam que o grau de escolaridade é de extrema importância, já que o cuidador deve seguir prescrições médicas, manusear medicamentos, entre outras tarefas. O estudo de Kawasaki e Diogo (2001), ao analisar o perfil do indivíduo que se oferece para cuidar de idosos em domicílio, no município de Campinas, observou que há um predomínio da baixa qualificação. Tais dados vêm ao encontro do presente estudo, cuja amostra não apresenta nenhum cuidador com curso superior.

A capacitação de recursos humanos especializados (profissionais) e o apoio para cuidados informais também é destaque entre as diretrizes da Política Nacional de Saúde do Idoso (PNSI), tratando-se de uma parceria entre o Ministério da Saúde e as Instituições de Ensino Superior (IES). Diogo (2004) descreve obstáculos nessa capacitação, tais como: escassez de conhecimento na área gerontogeriátria, a falta de experiência do corpo docente e a distância 
entre a prática e as discussões encaminhadas pelas IES. Geralmente, o conhecimento sobre atenção à população idosa está diluído em diversas disciplinas, na formação dos profissionais da saúde.

Born (2006) afirma que a emergência da ocupação do cuidador remunerado ou formal exigiu que critérios mínimos fossem estabelecidos para regularização dessa profissão. A Classificação Brasileira de Ocupações do Ministério do Trabalho e Emprego, segundo o autor, concebe que o cuidador formal deve ser submetido a treinamento específico, ministrado por instituição reconhecida e com conteúdo aprovado. Além das habilidades técnicas, são exigidas qualidades morais e éticas, como a capacidade de estabelecer vínculos de confiança, qualidades emocionais, físicas, intelectuais e de motivação.

b) Aspectos de rotina dos idosos na instituição

Tabela 1 - Frequência de relatos sobre cuidados gerais e práticas de lazer

\begin{tabular}{|c|c|c|}
\hline Categorias & 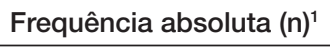 & Frequência relativa (\%) \\
\hline Ministrar medicação & 5 & $43,0 \%$ \\
\hline Higienização geral & 3 & $27,0 \%$ \\
\hline $\begin{array}{l}\text { Auxilio para práticas de } \\
\text { exercícios físicos no leito }\end{array}$ & 1 & $11,0 \%$ \\
\hline $\begin{array}{c}\text { Práticas de recreação na } \\
\text { própria instituição }\end{array}$ & 2 & $19,0 \%$ \\
\hline Total & 11 & $100,0 \%$ \\
\hline
\end{tabular}

1 - Os dados da frequência absoluta expressam a possibilidade de mais de uma resposta por participante.

Observa-se, de acordo com os relatos dos participantes apresentados na Tabela 1, que as atividades de rotina realizadas com os idosos na instituição estão relacionadas às atividades de vida diária (AVD). Um dos motivos que justifica a presença apenas desse tipo de atividade pode estar ligado à formação dos cuidadores e tarefas que os mesmos devem desempenhar na instituição. Para que houvesse a realização de outras práticas, que envolvessem fundamentalmente, aspectos sociais, intelectuais e emocionais, seria necessária a presença de outros profissionais nessa instituição, como é previsto em lei (BRASIL, 2007). 
Por outro lado, esses mesmos cuidadores poderiam contribuir com essa população por meio da realização de atividades com características sociocognitivas e socioculturais, favorecendo a preservação de aspectos importantes em relação à linguagem e à memória. Para tanto, deveriam ser instruídos, recebendo de modo efetivo, orientações para tal. O que se propõe aqui é que o profissional que fica diariamente com o idoso receba formação sobre diversos aspectos, preocupando-se com outras atividades e cuidados, além daquelas que são da área da enfermagem (BORN, 2006).

Observa-se que nem todos os cuidadores citaram as práticas de lazer, o que pode nos levar a inferir que não são muito frequentes. Além disso, essas são realizadas somente na instituição.

É consenso entre vários estudos, que essas práticas fornecem elementos essenciais para o processo de socialização e, portanto, ocupam um papel crucial na manutenção da qualidade de vida desses indivíduos. Na pesquisa de Gomes e Pinto (2006), os autores constataram que os interesses de lazer de idosos, são diversificados e contemplam atividades físico-esportivas, artísticas, sociais, manuais, intelectuais e turísticas. E mesmo assim, tem-se visto muito pouco em relação à rotina dessas práticas, como é o caso do presente estudo.

Amaral (2004) alerta para o fato de que o Estado tenha que assumir uma maior responsabilidade sobre as políticas públicas de lazer voltadas para essa população. Contudo, além disso, essa responsabilidade deve também ser compartilhada com a sociedade e a iniciativa privada, tomando-se o cuidado para que o lazer não seja visto apenas como mercadoria de consumo.

c) Aspectos sobre a rotina de alimentação dos idosos

Tabela 2 - Frequência de relatos sobre a percepção de fatores de risco para alterações de deglutição em idosos

\begin{tabular}{c|c|c}
\hline Categorias & Frequência absoluta $(\mathbf{n})^{2}$ & Frequência relativa (\%) $^{\text {Depressão }}$ \\
\hline $\begin{array}{c}\text { Acidente Vascular Encefálico } \\
\text { (AVE) }\end{array}$ & 3 & $15,0 \%$ \\
\hline Demência & 4 & $22,0 \%$ \\
\hline Problemas de comunicação & 2 & $6,0 \%$ \\
\hline Hipertensão & 2 & $10,0 \%$ \\
\hline Diabetes & 3 & $10,0 \%$ \\
\hline
\end{tabular}


(continuação)

\begin{tabular}{c|c|c}
\hline Mal de Alzheimer & 1 & $6,0 \%$ \\
\hline Doenças cardíacas & 2 & $10,0 \%$ \\
\hline Esclerose & 1 & $6,0 \%$ \\
\hline Total & $\mathbf{1 8}$ & $\mathbf{1 0 0 , 0 \%}$ \\
\hline
\end{tabular}

2 - Os dados da frequência absoluta expressam a possibilidade de mais de uma resposta por participante.

Observa-se na Tabela 2 que os cuidadores relataram vários aspectos patológicos como possíveis fatores de risco para alterações do processo de deglutição, dentre os quais podem ser destacados: Depressão (15\%); AVE (22\%); e Diabetes (15\%). Embora alguns desses fatores possam estar indiretamente relacionados com o processo de deglutição, os relatos foram de extrema coerência em relação ao processo de alimentação.

Sobre a depressão, todos os indivíduos com essa doença estão sujeitos ao uso de medicamentos, que segundo Souza et al. (2003) podem piorar ou melhorar os sinais da dificuldade de deglutição. Alguns medicamentos podem causar déficit cognitivo e secura das mucosas (xerostomia), cujo efeito fisiológico pode causar ou piorar a dificuldade de deglutição, ou ainda, induzir a uma esofagite. Além disso, a depressão retira o prazer que a alimentação pode fornecer ao indivíduo, alterando sobremaneira, a fase preparatória desse processo.

Filho, Gomes e Furkim (2000) também comentam sobre os efeitos dos medicamentos no Sistema Nervoso Central (SNC) e periférico ou muscular. Os efeitos causados são: depressão do nível de consciência (sedativos, hipnóticos e anticonvulsivantes), supressão da regulação central da deglutição (benzodiazepínicos) e desordens do movimento (neurolépticos e metoclopramida). Já o mecanismo de ação da disfagia, causada por medicamentos que atuam no sistema nervoso periférico, é o bloqueio da junção neuromuscular, que pode provocar fraqueza da musculatura da faringe. Dessa forma, mesmo que os cuidadores não tenham relatado tal associação, é possível estabelecêla, ainda que de modo indireto.

Nota-se também que os fatores de risco relatados estão relacionados às doenças crônicas, como, por exemplo, nos casos de Acidentes Vasculares Encefálicos (AVE). Para Magalhães e Bilton (2004), o AVE é uma das alterações neurológicas mais frequentes no envelhecimento que afetam o sistema sensório-motor-oral e comprometem a qualidade de vida. As sequelas do AVE podem ser variadas, sendo mais comuns as alterações locomotoras 
gerais, alterações de componentes motores da fala e alterações na dinâmica da deglutição (FORTES; FRANÇA; COSTA, 2004; FILHO; GOMES; FURKIM, 2000).

Cabe ressaltar que a hipertensão, também relatada pelos cuidadores, é uma das maiores responsáveis pelos casos de AVE (ROASCH, 2003). O Diabetes, também é indicado como fator de risco para alterações de deglutição, quando na verdade, o é para a hipertensão arterial. Interessante notar que os fatores de risco apontados são patologias e que nenhum cuidador referiu o envelhecimento como modificador da deglutição.

Há outros dados que também chamam a atenção por terem sido relatados, ainda que em menor frequência. É o caso de processos demenciais (6\%), ou seja, déficits cognitivos. A presença dessa característica pode, eventualmente, ocasionar junto a outros fatores, alterações de motricidade orofacial na população idosa. Com a diminuição das capacidades cognitivas, motoras e sensitivas, esses indivíduos tornam-se dependentes de outras pessoas para comer e beber. A partir disso, também podem ser observadas algumas alterações específicas de alimentação, tais como jogar comida fora; resistência a comer; mastigar sem parar; comer muito depressa ou devagar; esquecer de engolir; não sentar para comer; brincar com a comida; alteração da movimentação das estruturas orais; alteração do vedamento labial, dentre outras (MARCHESAN, 2003).

Nesses casos, os cuidadores precisam estar devidamente orientados, tanto em relação à presença desse diagnóstico, quanto às atitudes e procedimentos que deverão ser tomados durante a alimentação de indivíduos com quadros demenciais.

Tabela 3 - Frequência de relatos sobre o posicionamento dos idosos durante a alimentação

\begin{tabular}{c|c|c}
\hline Descrições dos cuidadores & Frequência absoluta (n) & Frequência relativa (\%) \\
\hline $\begin{array}{c}\text { Deixa o idoso sentado; } \\
\text { eleva a cabeça; }\end{array}$ & 3 & $60,0 \%$ \\
\hline $\begin{array}{c}\text { Deixa o idoso sentado } \\
\text { ou deitado; }\end{array}$ & 2 & $40,0 \%$ \\
\hline Total & $\mathbf{5}$ & $\mathbf{1 0 0 , 0 \%}$ \\
\hline
\end{tabular}

Sobre o posicionamento dos idosos durante a alimentação, observa-se que $3(60 \%)$ cuidadores relataram adoção de posições corretas, descrevendo principalmente posições sentadas com elevação de tronco e apoio da coluna cervical com travesseiros. Por outro lado, $2(40 \%)$ relataram que alimentam 
os idosos sentados e também deitados. Sabe-se que a posição deitada não auxilia o processo de deglutição, pois o alinhamento anatômico correto facilita a passagem do alimento pela faringe e esôfago com menor comprometimento da respiração e deglutição (BRAUER; FRAME, 2001).

Ainda sobre esse posicionamento, é viável que se mantenha a cabeça elevada e seja colocado um travesseiro de apoio na parte inferior das costas e outros adicionais dos lados e embaixo dos joelhos (para evitar que o indivíduo escorregue). O ideal é que a cabeceira da cama seja elevada em torno de 70 a $90^{\circ}$. Se essa alimentação ocorrer na cadeira, deve-se manter o idoso sentado em posição ereta e usar travesseiro ou toalhas enroladas, caso ele venha a pender para um dos lados. Além disso, o cuidador deve se certificar de que a pélvis esteja encostada detrás da cadeira, a fim de que o indivíduo não escorregue e, por fim, deve evitar a hiperextensão do pescoço, de modo que a cabeça fique pendendo ligeiramente para a frente, de forma a proteger as vias aéreas (BRAUER; FRAME, 2001; FURKIN, 1999).

Tabela 4 - Frequência de relatos acerca da prática de higienização oral após alimentação

\begin{tabular}{c|c|c}
\hline Categorias & Frequência absoluta $(\mathbf{n})$ & Frequência relativa (\%) \\
\hline Realização & 0 & $00,0 \%$ \\
\hline Não realização & 5 & $100,0 \%$ \\
\hline Total & $\mathbf{5}$ & $\mathbf{1 0 0 , 0 \%}$ \\
\hline
\end{tabular}

Sobre a higienização oral após a alimentação, os cuidadores foram unânimes em relatar que não realizam tal prática com os idosos, ficando tal cuidado sob responsabilidade destes.

No idoso, os cuidados com a cavidade oral se tornam cada vez mais necessários, pois a boca está ligada intimamente com um dos momentos mais prazerosos dos idosos, que é a alimentação. Manter a integridade e função da saúde bucal está também relacionado com a estética, o conforto, a habilidade para mastigar, sentir sabor e falar (ESCARPA, 1990; FURKIN, 1999).

Essa higienização deve ser realizada todos os dias, ao acordar, após todas as refeições e no momento de se repousar. Caso o idoso não tenha condições próprias de realizá-la sozinho, a mesma deverá ser feita por um cuidador.

Além de garantir essa integridade, a higienização oral, feita de maneira satisfatória, pode prevenir o surgimento de futuras doenças. Essas, ocasionadas por bactérias que se encontram na cavidade oral do indivíduo e que 
podem até mesmo atingir vias respiratórias, digestivas, entre outras (RIEGEL, 2006).

Segundo Filho, Gomes e Furkim (2000) a prática da higienização oral em indivíduos que apresentam uma salivação excessiva, pode ser realizada por meio da aspiração da saliva e dos restos alimentares da cavidade oral com um aspirador acoplado a uma sonda. A ação mecânica da escovação dos dentes, o uso de fraldas e/ou gazes, também se constituem como principais estratégias para realizar tal limpeza, evitando assim, o surgimento de placas bacterianas e também o aparecimento de infecções do trato respiratório.

\section{Considerações finais}

A grande maioria da população geriátrica institucionalizada possui uma ou mais doenças crônicas. Vimos que diversas patologias e medicamentos, ou até mesmo o avanço da idade, podem modificar o processo de deglutição. Em nosso estudo, com foco para a discussão do perfil do cuidador de idosos institucionalizados para auxiliar na tarefa de alimentação, verificou-se que os cuidadores não relataram fatores de risco para essas modificações, embora tenham referido doenças relacionadas à disfagia.

A respeito do conhecimento de técnicas que facilitam a deglutição, chamou-nos a atenção que a prática da higienização oral não era realizada pelos cuidadores e estes não fizeram nenhuma referência acerca de utensílios, volumes e consistências dos alimentos. Sabe-se que mudanças relativas a esses aspectos podem favorecer a deglutição, quando orientadas por um profissional especializado. Além disso, manobras posturais e de proteção de vias áreas podem ser realizadas para evitar episódios de engasgos (FURKIN, 1999).

Esses dados nos remetem às reflexões atuais sobre a formação do cuidador para melhor adaptação da alimentação no idoso. Nesse contexto, o fonoaudiólogo, que tem um papel fundamental no diagnóstico e tratamento das disfagias (distúrbios da deglutição), poderia contribuir com essa formação.

Avançando em relação às práticas, nas atuais políticas públicas, encontramos propostas voltadas para o cuidado do idoso, demonstradas pelo reconhecimento do cuidador formal pela Classificação Brasileira de Ocupações do Ministério do Trabalho e Emprego e pelo Estatuto do Idoso. Contribuindo com esses avanços, seria necessário que todas as profissões de saúde se integrassem mais à temática. Essa integração pode ser concretizada pela promoção de discussões que perpassem pelos conteúdos já previstos nas grades 
curriculares e também através de um maior conhecimento da prática relativa a esse cuidado, tanto por docentes de IES, quanto pelos discentes.

Os resultados apresentados indicam ainda a necessidade de realização de estudos com métodos semelhantes com amostras mais significativas. Além disso, sugerem a continuidade de pesquisas com caráter interdisciplinar.

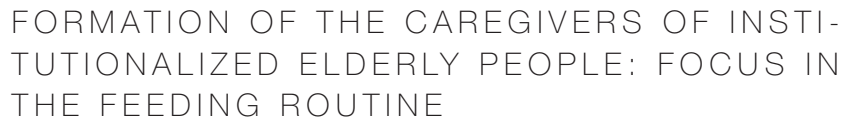

abstract

This study aims to argue about the profile of the caregivers of aged people to assist in the feeding task. The sample was composed by interviews with five professionals of the health, operating in the homes for the aged. The caregivers reported risk factors for changes in swallowing and absence of performing the oral hygiene of the elderly people. The results show the necessity of reflection about the formation of caregivers to assist in feeding the elderly. The speech therapist can guide the caregivers to facilitate the swallowing of the elderly in ILP

keywords

Caregivers. Homes for the Aged. Feeding. Formation.

\section{referências}

BORN, Tomiko. Quem vai cuidar de mim quando eu ficar velha? Revista Kairós: São Paulo, v. 4, n. 2, p. 135-148, 2001.

BORN, Tomiko. A. Formação de cuidadores: acompanhamento e avaliação. Seminário Velhice fragilizada. São Paulo, 2006, 15f. Disponível em: mww.sescsp.org.br/sesc/images/upload/conferencias/366rtf. Acesso em 30 de mar. de 2006.

BRASIL. Ministério da Saúde. Estatuto do ldoso/Ministério da Saúde. 2. ed., $1^{a}$ reimpr. Brasília, 2007. Disponível em: unw.bvsms.saude.gov.br/bvs/publicacao, acesso em 30 de mar. de 2006.

BRAUER, Carla; FRAME, Dyanne. Manual de Disfagia. Carapicuíba, Pró-Fono, 2001.

CERQUeIRA, Ana Teresa A. R. OLIVEIRA, Nair I. L. Programa de apoio a cuidadores: uma ação terapêutica e preventiva na atenção à saúde dos idosos. Revista de Psicologia da USP, São Paulo, v. 13, n. 1, p. 133-150, 2002. 
DIOGO, Maria José. Formação de recursos humanos na área da saúde do idoso. Revista Latino Americana de Enfermagem, v. 12, n. 2, p. 280-282, 2004.

FlLHO, Evaldo D. M. F.; GOMES, Guilherme; FURK
do paciente com disfagia. São Paulo: Lovise, 2000.

FORTES, Vera Lucia F.; FRANÇA, Renata M.; COSTA, Gerson Luís. O Idoso com Acidente Vascular Cerebral (AVC) Isquêmico Agudo: Vivenciando o Cuidado. Revista Brasileira de Ciências do Envelhecimento Humano, Passo Fundo, v. 1, n. 2, p. 22-29, 2004.

FREIRE JR, Renato C.; TAVARES, Maria de Fátima L. A saúde sob o olhar do idoso institucionalizado: conhecendo e valorizando sua opinião. Revista interface: Comunicação, Saúde, Educação, Botucatu, v. 9, n. 16, p. 147-58, 2004

FURKIM, Ana Maria. Fonoterapia nas Disfagias Orofaríngeas. In: FURKIM, Ana Maria; SANTINI, Celia S. (Org.). Disfagias Orofaringeas. Carapicuíba: Pró-Fono, p. 229-258, 1999.

GOLDFARB, Delia C. Velhices fragilizadas: espaços e ações preventivas. In: Velhices: reflexões contemporâneas. São Paulo, Editora PUC-SESC, 2006.

GOMES, Christianne L.; PINTO, Gabriela B. O lazer na velhice: reflexão sobre as experiências de um grupo de idosos. Revista Kairós, São Paulo, v. 9, n. 2, p. 113-133, 2006

GONÇALVES, Luciana O. Cuidadores primários familiares dos idosos atendidos na Clínica Escola de Fisioterapia da Universidade do Vale do Itajaí - UNIVALI. [Dissertação]. Florianópolis: Programa de Pós-Graduação em Engenharia de Produção/UFSC. 2002.

JALES, Mariana A.; CABRAL, Rodrigo R.; SILVA, Hilton J.; CUNHA, Daniel A. C. Características do sistema estomatognático em idosos: diferenças entre instituição pública e privada, Revista CEFAC, São Paulo, v. 7, n. 2, p. 178-87, 2005

KARSCH, Úrsula. Idosos dependentes: famílias e cuidadores. Cadernos de Saúde Pú blica, Rio de Janeiro, v. 19, n. 3, p. 861-863, 2003

KAWASAKI, Kozue; DIOGO, Maria José. Assistência domiciliária ao idoso: perfil do cuidador formal - parte I. Revista da Escola de Enfermagem da USP. Ribeirão Preto, v. 35 n. 3, p. 257-264, 2001.

LIMA-COSTA, Maria Fernanda; BARRETO, Sandhi M. Tipos de estudos epidemiológicos: conceitos básicos e aplicações na área do envelhecimento. Epidemiologia e Senviços de Saúde, v. 12, n. 4, p. 189-201, 2003.

MARCHESAN, Irene Q. O que se considera normal na deglutição. In: JACOBI, Juliana da S.; LEVY, Débora S.; SILVA, Luciano M. C. Disfagia: Avaliação e Tratamento. Rio de Janeiro: Revinter, p. 3-17, 2003

MARCHESAN, Irene Queiroz. Alterações de fala de origem musculoesquelética. In: FERREIRA, Léslie P.; BEFI-LOPES, Débora M.; LIMONGI, Sueli C. O. Tratado de Fonoaudiologia, São Paulo: Roca, p. 292-303, 2004.

MARTINS, Maristela S.; MASSAROLLO, Maria Cristina K. B. Mudanças na assistência ao idoso após promulgação do Estatuto do Idoso segundo profissionais de hospita geriátrico. Revista da Escola de Enfemagem da USP, Ribeirão Preto, v. 42, n. 1, p. 26-33, 2008.

MENDONÇA, José Alexandre; MARQUES NETO, João Francisco. Qualidade de vida do idoso institucionalizado frente aos grupos de afecções crônicas. Revista de Ciências Médicas de Campinas, Campinas, v. 12, n. 4, p. 299-306, 2003

OLIVEIRA, Jáima P.; SANTOS, Tallita G. História de vida e habilidades comunicativas de idosos institucionalizados. A Terceira ldade. São Paulo, v. 19, n. 2, p. 50-63, 2008. 
Recebido: 13/03/2010
OMS - Organização mundial de Saúde. CIF: Classificação Internacional de Funcionalidade, Incapacidade e Saúde. Centro Colaborador da Organização Mundial da Saúde para a Família de Classificações Internacionais. Tradução de Cássia Maria Buchalla. São Paulo: EDUSP, 2003

RAMOS, Luiz; PERRACINI, Monica; ROSA, Tereza; KALACHE, Alex. Significance and management of disability among urban elderly residents in Brazil. Joumal of Cross-Cultural Gerontology, New York, v. 8, n. 4, p. 313-323, 1993

RAMOS, Luiz Roberto. Fatores determinantes do envelhecimento saudável em idosos residentes em centro urbano: Projeto Epidoso. São Paulo, Cademos de Saúde Pública, Rio de Janeiro, v. 19, n. 3, p. 793-798, 2003.

RIEGEL, Rafael B. Alterações bucais em idosos - artigos em odontologia: disponível em: http://muw.drgate.com.br/index.php?option=com. Acesso em: 30 de mar. de 2006.

ROCKLAND. Adriano; BORBA, Júlio. Primeiros passos na Fonoaudiologia - Conhecer para intervir nas patologias, distúrbios e exames fonoaudiológicos, São José dos Campos: Pulso Editorial, 2006

SALIBA, Nemre A.; MOIMAZ, Suzely A. S.; MARQUES, Jeidson A. M.; PRADO, Rosana L. Perfil de cuidadores de idosos e percepção sobre saúde bucal. Revista interface: Comunicação, Saúde, Educação, Botucatu, v. 11, n. 21, p. 39-50, 2007.

TEIXEIRA, Ilka Nicéia O.; NERI, Anita L. A fragilidade no envelhecimento: fenômeno multidimensional, multideterminado e evolutivo. In: FREITAS, Elizabete V.; PY, Lígia; CANCADO, Flávio A. X.; GORZONI, Milton Luiz. Tratado de Geriatria e Gerontologia, Rio de Janeiro: Guanabara-Koogan, p. 1102-1109, 2006 